\title{
A Systematic Review of Interventions to Reduce Problematic Substance Use Among Transgender Individuals: A Call to Action
}

\author{
Tiffany R. Glynn ${ }^{1,2, *}$ and Jacob J. van den Berg ${ }^{1,3,4}$
}

\begin{abstract}
Persons who are transgender (i.e., individuals who are assigned one sex at birth, but who do not identify with that sex) are at elevated risk for developing problematic substance use. Recent studies indicate that transgender persons have high rates of alcohol use, illicit drug use, and nonmedical use of prescription drugs and evince more severe misuse of these substances compared with nontransgender individuals. Despite the high rates of substance use among transgender persons and the multiple conceptual and narrative recommendations for substance use treatments, there is a lack of consensus or awareness of empirically tested interventions and programs effective for this population. Thus, it is critical to examine current substance use interventions for transgender individuals to identify gaps in the field and to immediately put forth efforts to reduce problematic substance use. This systematic review is the first to attempt a comprehensive identification and synthesis of the available evidence on interventions for reducing problematic substance use among transgender persons. Reflective of the state of the field regarding transgender care for substance use, we found a deficiency of studies to include in this systematic review $(n=2)$. Perhaps the most important conclusion of this review is that welldesigned, theoretically informed culturally sensitive research focused on developing and rigorously testing interventions for substance use among transgender individuals is alarmingly scarce. This review discusses barriers to intervention design and synthesizes treatment recommendations for future work.
\end{abstract}

Keywords: alcohol; drug use; substance use interventions; substance use treatment; systematic review; transgender individuals

\section{Introduction}

Persons who are transgender (i.e., individuals who are assigned one sex at birth, but who do not identify with that sex) are at elevated risk for developing problematic substance use. ${ }^{1}$ Problematic substance use can be considered the umbrella term to encompass the multiple facets and terminology used by the World Health Organization, ${ }^{2}$ the National Associations for National Institute on Alcohol Abuse and Alcoholism (NIAAA), ${ }^{3}$ and the
National Institute on Drug Abuse ${ }^{4}$ for using alcohol or other drugs in excess. For example, engaging in binge drinking (e.g., defined as four to five or more drinks consumed on one occasion at least once in a 2 -week period), using prescription medication(s) more than prescribed or not with a doctor's prescription, or regular drug use (e.g., opioids, amphetamines, or cocaine). It also encompasses experiencing substance-related problems (e.g., motor vehicle accident from driving under the

\footnotetext{
${ }^{1}$ Department of Behavioral and Social Sciences, Brown University School of Public Health, Providence, Rhode Island.

${ }^{2}$ Department of Psychology, University of Miami, Coral Gables, Florida.

${ }^{3}$ Department of Medicine, The Warren Alpert Medical School of Brown University, Providence, Rhode Island.

${ }^{4}$ Division of Infectious Diseases, The Miriam Hospital, Providence, Rhode Island.

*Address correspondence to: Tiffany R. Glynn, MS, Department of Psychology, University of Miami Miller School of Medicine, Clinical Research Building, 1120 Northwest 14th Street, Suite 787, Miami, FL 33136, E-mail: tiffany.glynn@miami.edu

(c) Tiffany R. Glynn and Jacob J. van den Berg, 2017; Published by Mary Ann Liebert, Inc. This Open Access article is distributed under the terms of the Creative Commons License (http://creativecommons.org/licenses/by/4.0), which permits unrestricted use, distribution, and reproduction in any medium, provided the original work is properly credited.
} 
influence) or having any substance use disorder diagnoses as defined by the Diagnostic and Statistical Manual of Mental Disorders. ${ }^{5}$ Recent studies indicate that transgender individuals have high rates of alcohol use (estimates up to $72 \%$ ), marijuana (estimates up to $71 \%$ ), other illicit drug use (estimates up to $34 \%$ [including intravenous drug use]), and nonmedical use of prescription drugs (estimates up to $26.5 \%$ ) and evince more severe misuse of these substances compared with nontransgender persons. ${ }^{6-14}$

In examining this disparity between transgender and nontransgender individuals, studies have noted associations between trans-specific discrimination (e.g., transphobia, gender abuse, and religious abuse) and substance use. ${ }^{15-20}$ These findings support the Minority Stress Model, ${ }^{21}$ which posits that prolonged exposure to prejudice and discrimination experienced by members of minority and marginalized groups is associated with adverse psychological outcomes and health risk behaviors such as substance use.

Other scholars investigating problematic substance use among transgender populations have done so in the context of HIV risk due to the high prevalence of HIV among this group, especially transgender women, which has been well-documented across numerous studies. ${ }^{22-24}$ A syndemic framework ${ }^{25}$ has been used to contextualize the HIV epidemic among transgender persons positing that HIV risk is rooted in multiple co-occurring public health problems, including psychological issues, violence, discrimination, and substance use, which act synergistically to drive sexual risk behaviors. ${ }^{24,26-29}$ This syndemic among transgender communities can be conceptualized as intertwining epidemics (including problematic substance use) that act multiplicatively to mutually reinforce and predict HIV risk. For example, HIV-related risk behaviors (e.g., condomless sex) have been shown to be specifically exacerbated by substance use, ${ }^{30}$ especially among transgender communities, ${ }^{12,15,31-35}$ demonstrating that solely focusing on sexual behaviors for reducing the transmission of HIV may not be sufficient without also targeting problematic substance use. Given the high prevalence of problematic substance use among transgender persons, it is important for researchers, public health professionals, and policy makers to understand how problematic substance use contributes to this syndemic to intervene and reduce behavioral risks. ${ }^{23,36}$

In response to these substance use rates and the association with HIV, scholars dedicated to lesbian, gay, bisexual, and transgender (LGBT) health have called for substance use interventions specifically for transgender populations. More than 15 years ago, Lombardi and van Servellen ${ }^{37}$ proposed the need for specialized substance use treatments for LGBT people to remedy the deficiencies (e.g., discrimination, biases) found in general treatment programs. Following this, the Substance Abuse and Mental Health Services Administration (SAMHSA) released a policy acknowledging the special needs of these populations with the aim to make researchers and treatment providers aware of LGBT-specific issues in substance use treatment. ${ }^{38}$ Stevens ${ }^{39}$ furthered this call for specialty substance use treatment for LGBT individuals, but acknowledged the barrier that specialized care is difficult to find; thus, cultural sensitivity training for providers and staff in general care is still urgently needed today.

More specific to transgender individuals, the Transgender Substance Abuse Treatment Policy Group of the San Francisco Lesbian, Gay, Bisexual, and Transgender Task Force ${ }^{40}$ provided guidelines regarding cultural sensitivity and program design for substance use treatment programs to adopt in order to facilitate better care for transgender persons. The need for specialized programs designed to meet the unique needs of transgender individuals (e.g., addressing facets of gender affirmation, minority stress, or the syndemic framework regarding co-occurring problems) and cultural competency among treatment providers continues to be reiterated in the burgeoning literature in this area. ${ }^{13,17,39,41,42}$ Taken together, there are two paths to improve substance use intervention outcomes for transgender individuals: programs designed specifically for transgender persons and revamping general substance use programs to be sensitive to transgender needs within their more broad treatment.

Although this call for specific attention to address problematic substance use among transgender individuals has been made, there has been no systematic investigation into the current state of interventions for the transgender community. The majority of systematic reviews and meta-analyses examining substance use interventions broadly have focused on general adult or adolescent populations ${ }^{43-49}$ and have not been dedicated to specific subpopulations. This could be a reflection of the fact that evidence-based practices for problematic substance use are mainly developed and tested for the majority population. This general focus disregards the heterogeneity in specific subpopulations, especially for those at higher risk for problematic substance use such as those in the transgender community. 
Substance use programs that have been adapted or that have added elements to address issues specific to people's unique parameters (e.g., race/ethnicity, age, and sexual orientation) have been shown to be effective in helping facilitate harm reduction and reduced use of substances, ${ }^{50-53}$ indicating a need to utilize culturally sensitive interventions and programs when available.

Despite the high rates of substance use in transgender individuals and the multiple conceptual and narrative recommendations for substance use treatments, there is a lack of consensus or awareness of empirically tested interventions and programs effective for transgender persons. Given the political and social climate of the transgender community gaining more visibility and greater concentrated efforts promoting their health and civil rights, it is critical to review current substance use interventions for transgender individuals to identify gaps in the field and to immediately put forth efforts to reduce problematic substance use. Moreover, due to the multiplicative nature of HIV risk and substance use in transgender communities, a review would be beneficial because knowing the most effective ways of intervening on problematic substance use may reduce HIV incidence.

This is the first known systematic review investigating interventions for problematic substance use for transgender individuals. The aims of this study were to (1) explore the literature for substance use interventions for transgender individuals in order to examine the current state of the field; (2) describe the characteristics of included studies; (3) summarize the findings across included studies to examine efficacy and inform future work; and (4) synthesize treatment recommendations from the literature to advise intervention development.

\section{Methods}

Study selection

This review followed an a priori systematic review protocol to reduce bias of selection and reporting of studies. Our a priori protocol identified search parameters, including the population of interest, the types of interventions to include, if interventions needed to have any control or comparison group, and defining the outcomes of interest.

Population. For population eligibility, included studies were those that targeted intervention activities specifically toward transgender people. Studies that targeted transgender individuals in addition to other populations were included if they disaggregated findings for transgender participants. Included transgender populations were not restricted to the United States and could be from any country as long as there was an English-language translation of the article.

Intervention. To truly examine all available interventions in the literature, any type of study design evaluating an intervention for reducing problematic substance use was included. Eligible interventions could take a psychosocial, behavioral, structural, or medical approach and could also address other comorbid issues, so long as the intervention had a clear component addressing substance use specifically.

Comparison group. To ensure greatest sensitivity, any type of comparison group or those with no comparison group were included.

Outcomes. Problem substance use was interpreted broadly and included studies designed to target those using substances beyond recommended and/or medical guidelines (e.g., weekly drinking levels as defined by the NIAAA; using prescription medication more than prescribed or not with a doctor's prescription), those experiencing substance-related problems, or those with a substance use disorder diagnoses. The primary outcomes of interest in this review were changes in substance use, as measured by biological markers or self-report.

\section{Search strategy}

Five electronic databases were searched (PubMed, PsychINFO, LGBT Life, CINHAL, and CENTRAL) for studies published through July 2016 (see Fig. 1 for the Preferred Reporting Items for Systematic Reviews and Meta-Analyses [PRISMA] ${ }^{54}$ flowchart of systematic review). The search strategy included free-text terms relating to populations and outcomes of interest to be able to use the same search across databases. The search terms for the electronic databases for the outcome of interest, changes in substance use, were derived from past Cochrane Reviews, ${ }^{55,56}$ illicit drugs measured in the Structured Clinical Interview for Diagnostic and Statistical Manual of Mental Disorders (DSM),${ }^{57}$ and a past review regarding alcohol interventions. ${ }^{58}$ To specify the population of interest, we used search terms for transgender individuals derived from a past review $^{59}$ and also additional terms derived from consultation with a published and experienced researcher in the field of transgender health. To increase sensitivity and given the wide criteria for any type of 


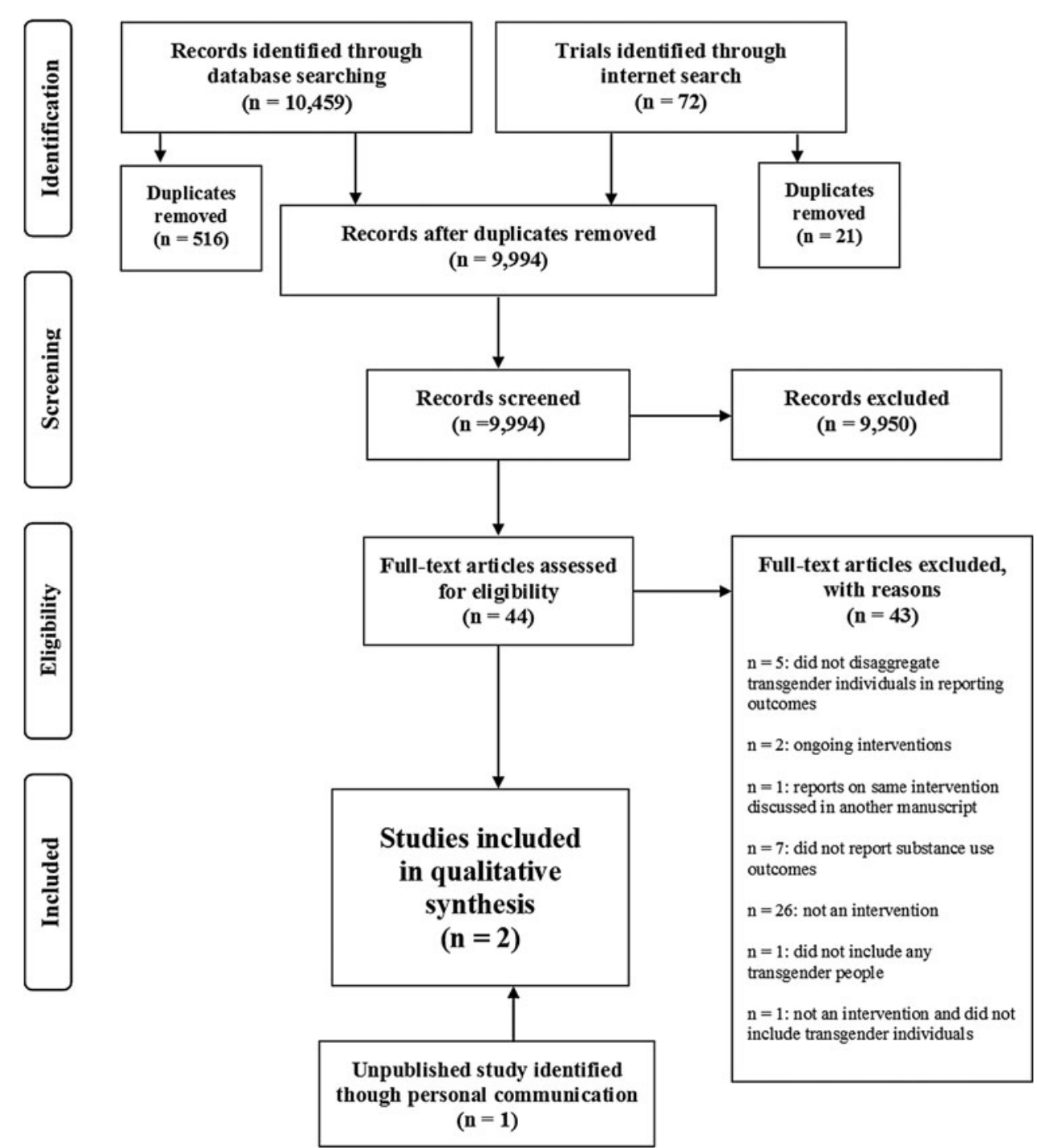

FIG. 1. Preferred Reporting Items for Systematic Reviews and Meta-Analyses (PRISMA) flowchart of systematic review.

intervention and any or no comparison group, no search filters were used for intervention type (see Appendix A1 for all search terms). In addition to the electronic databases, ongoing and completed clinical trials were searched through Internet searches (see Appendix A2 for included websites and search terms). This was done to capture any completed clinical trial not found in the database search and to also be able to find and report on any current interventions being conducted. Although ongoing interventions cannot be included in the review due to lack of outcomes, acknowledging this work adds to the understanding of the current state of the field. The results of the five sets of electronic searches were collated $(n=10,459)$ into a single database and duplicates removed. Internet searches were conducted separately $(n=72)$ and duplicates removed. Titles and abstracts of all identified records after duplication removal $(n=9,994)$ were initially screened by the first author to exclude citations that were not relevant. A total of 44 records met preliminary inclusion criteria based on information presented in the abstract or title. Full texts for all preliminary eligible records were retrieved, which were then thoroughly read for further eligibility separately by both 
authors and then discussed to reach a consensus based on the a priori criteria. This produced the final list of included studies $(n=1)$. Due to the lack of studies found, and to produce a thorough review, academic experts in transgender health were contacted $(n=10)$ and asked to add any additional studies they were aware of. Five experts responded and this personal communication produced one more intervention to include in the current review that has been presented at a national conference with the primary outcome article in preparation, thus making the final list of included studies $n=2$.

\section{Data extraction}

For the included studies, data were extracted by the first author and included details about study participants, inclusion criteria, intervention design, and results (Table 1).

\section{Results}

The majority of studies reviewed were not an intervention $(n=26)$, and others discussed substance use interventions for transgender individuals, but did not report any outcomes $(n=7)$. There were some studies that identified transgender individuals in their samples, but did not report separate outcomes for them $(n=5)$, so these studies were excluded. Another study $(n=1)$ did not include any transgender people, and another $(n=1)$ was not an intervention nor did it include transgender people. One article discussed an intervention that was already reported on in another reviewed article (see Table 2 for summary of exclusions). Although excluded, many of these studies had great value in informing the discussion of the results. Two ongoing interventions were found (Table 3), so effectiveness cannot be currently discussed, but these should be acknowledged to examine outcomes in the future.

\section{Characteristics of included studies}

Of the 44 studies screened for inclusion and after personal communication with academic transgender health experts, one cohort study published in $2005^{60}$ and one presented at a national conference and unpublished randomized control trial (RCT) ${ }^{61}$ were selected for this systematic review, reporting data from 109 transgender participants and 114 transgender participants, respectively (Table 1).

\section{Intervention design and content}

Both the Transgender Resources and Neighborhood Space (TRANS) program and the Transgender Empow- erment and Motivational Interviewing (TEAM-I) study were studies from the same research group and aimed to reduce HIV risk and substance use among transgender women in San Francisco, with the latter focusing on African American women and Latinas.

TRANS program. The intervention was designed as 18 one-hour group workshops, held weekly, organized into three domains: (1) sex, relationships, and health; (2) reducing drug use and improving coping skills; and (3) general life needs. Individuals were eligible to be in this study if they were transgender women aged 18 years or older residing or working in San Francisco. These sessions were conducted by health educators (all of whom were transgender women) in both English and Spanish. The health educators used multiple group facilitation techniques such as interactive discussions, personal expression exercise, videos and media, and guest lectures. The transgender women were encouraged to finish the workshops at their own pace, as opposed to following a strict schedule (average time to complete 10 sessions was 6 weeks). Nemoto et al. ${ }^{60}$ also noted that the women were encouraged to socialize at the TRANS program site, which provided a living room area and shower facility. A resource closet with donated clothing and accessories free to those in need was also provided to create a safe and culturally sensitive space for participants to engage with the intervention. Participants were asked to attend at least 10 of the 18 workshops to be considered program graduates. Preintervention, the women were asked to complete assessments on sexual behaviors, substance use, attitudes toward substance abuse treatment, HIV knowledge, depression, selfesteem, and transgender community involvement. If considered a graduate of the program (i.e., completing at least 10 workshops), participants were then asked to complete postintervention assessments of the same outcomes within 2 weeks of completion.

TEAM-I. This intervention was designed as an RCT specifically for transgender African American women and Latinas using three group conditions: (1) motivational enhancement intervention (MEI); (2) brief individualized health promotion education (BI); and (3) the control condition. Eligibility criteria included 18 years or older, African American or Latina, transgender woman, living or working in the San Francisco area, and reported drinking alcohol almost every day or using drugs more than 3 days a week in the past 6 months. All participants completed outcome assessments at 

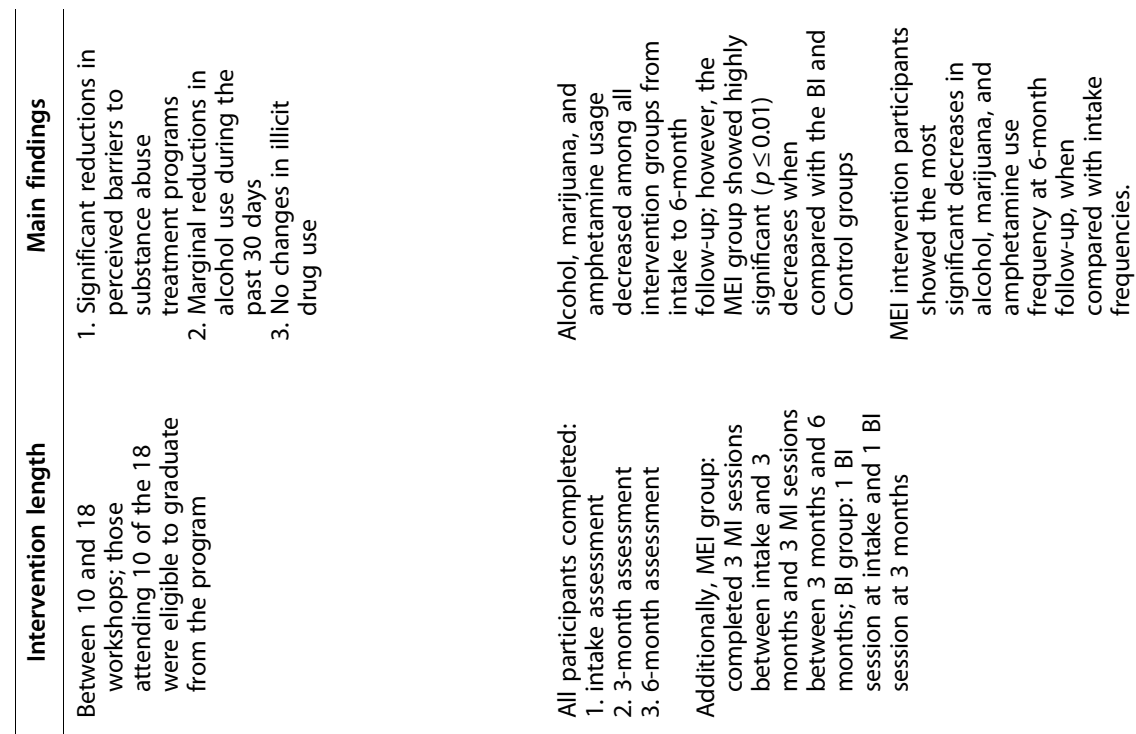

$$
\text { - }
$$

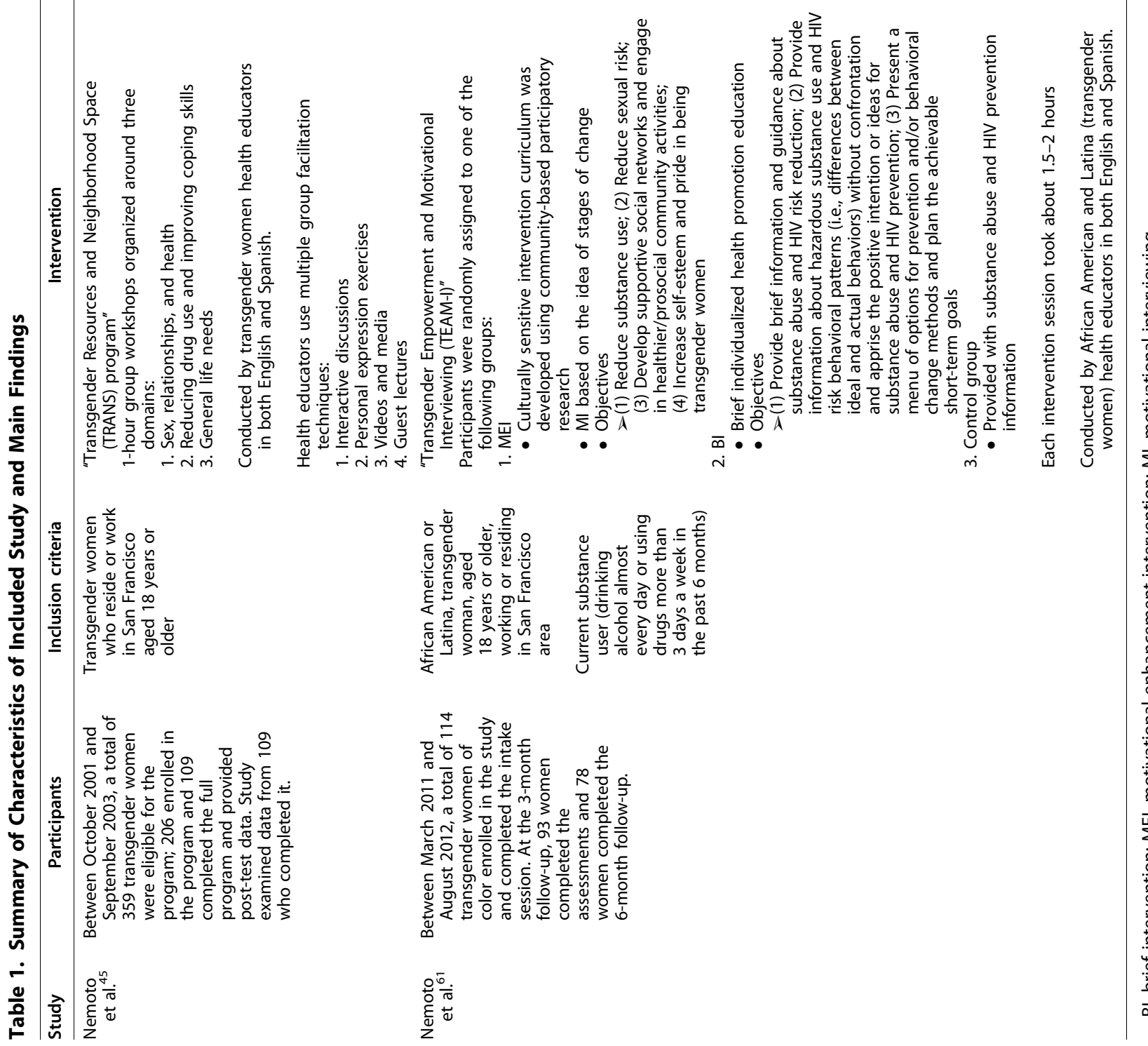


Table 2. Reviewed Full-Texts and Reasons for Exclusion

\begin{tabular}{|c|c|}
\hline Author & Reasons excluded \\
\hline Brown et al. $^{70}$ & Not an intervention \\
\hline Dunckley $^{86}$ & Not an intervention \\
\hline Finlon $^{87^{\circ}}$ & Not an intervention \\
\hline Gelaude et al. ${ }^{88}$ & Not an intervention \\
\hline Gilbert et al. ${ }^{89}$ & Not an intervention \\
\hline Heck et al. ${ }^{90}$ & Not an intervention \\
\hline Hellman et al. ${ }^{91}$ & Not an intervention \\
\hline Jordan $^{92}$ & Not an intervention \\
\hline Keuroghlian et al. ${ }^{65}$ & Not an intervention \\
\hline Klein and Ross ${ }^{93}$ & Not an intervention \\
\hline Krishnan et al. ${ }^{94}$ & Not an intervention \\
\hline Lyons et al. ${ }^{71}$ & Not an intervention \\
\hline Medeiros et al. ${ }^{95}$ & Not an intervention \\
\hline Nuttbrook et al. ${ }^{84}$ & Not an intervention \\
\hline Patel et al. ${ }^{96}$ & Not an intervention \\
\hline Pettifor et al. ${ }^{97}$ & Not an intervention \\
\hline Polak et al. ${ }^{81}$ & Not an intervention \\
\hline Ritter $^{98}$ & Not an intervention \\
\hline Senreich $^{99}$ & Not an intervention \\
\hline Senreich ${ }^{100}$ & Not an intervention \\
\hline Senreich $^{72}$ & Not an intervention \\
\hline Silvestre ${ }^{101}$ & Not an intervention \\
\hline Stevens ${ }^{39}$ & Not an intervention \\
\hline Warren et al. ${ }^{102}$ & Not an intervention \\
\hline Wolf et al. ${ }^{13}$ & Not an intervention \\
\hline Ziegler $^{103}$ & Not an intervention \\
\hline Goldbach et al. $^{104}$ & Did not report substance use outcomes \\
\hline Goyal et al. ${ }^{105}$ & Did not report substance use outcomes \\
\hline Hicks $^{106}$ & Did not report substance use outcomes \\
\hline Maguen et al. ${ }^{107}$ & Did not report substance use outcomes \\
\hline Oggins $^{76}$ & Did not report substance use outcomes \\
\hline Reback et al. ${ }^{24}$ & Did not report substance use outcomes \\
\hline Rebeck et al. ${ }^{108}$ & Did not report substance use outcomes \\
\hline Harris et al. $^{79}$ & $\begin{array}{l}\text { Did not report separate outcomes } \\
\text { for transgender individuals }\end{array}$ \\
\hline Maxwell $^{109}$ & $\begin{array}{l}\text { Did not report separate outcomes } \\
\text { for transgender individuals }\end{array}$ \\
\hline Rowan $^{110}$ & $\begin{array}{l}\text { Did not report separate outcomes } \\
\text { for transgender individuals }\end{array}$ \\
\hline Schwinn et al. ${ }^{111}$ & $\begin{array}{l}\text { Did not report separate outcomes } \\
\text { for transgender individuals }\end{array}$ \\
\hline Yu et al. ${ }^{112}$ & $\begin{array}{l}\text { Did not report separate outcomes } \\
\text { for transgender individuals }\end{array}$ \\
\hline Senreich ${ }^{113}$ & Did not include transgender individuals \\
\hline Allison et al. ${ }^{114}$ & $\begin{array}{l}\text { Not an intervention, nor did it include } \\
\text { transgender individuals }\end{array}$ \\
\hline Nemoto et al. ${ }^{78}$ & $\begin{array}{l}\text { Intervention was already reported } \\
\text { on in another reviewed article }\end{array}$ \\
\hline
\end{tabular}

an intake session, a 3-month follow-up session, and a final 6-month follow-up session. Additionally, the MEI group completed one MEI session at intake and two more before the 3-month assessment, then one at the 3-month assessment, and the final two MEI sessions before the 6-month assessment. The BI group completed one BI session at intake and one BI session at the 3-month session. The control group was asked to only complete the assessment sessions. The MEI protocol and aims were designed using community-based participatory research with a community advisory board comprising individuals from the San Francisco
Department of Public Health, community-based organizations serving the transgender community, and transgender community members (see Table 1 for details about each condition). As in the other included study, these sessions were conducted by health educators (all of whom were transgender women) in both English and Spanish. However, in contrast to the TRANS workshops delivered in groups, all sessions were delivered individually.

\section{Summary of intervention treatment effects}

TRANS program. Participant baseline levels of any alcohol use and any illicit drug use were $57.3 \%$ and $46.3 \%$, respectively. In regard to substance use outcome differences between pre- and postassessment, findings indicated significant reductions in perceived barriers to substance abuse treatment programs $(p=0.001)$; marginal reductions in alcohol use during the past 30 days $(p=0.06)$; and no changes in illicit drug use. The authors of the intervention noted that perhaps the marginally significant results would have been significant if a longer time lag had been implemented for administering follow-up surveys. In addition, longer time before follow-up might have allowed for more noteworthy behavioral changes in substance use. Another limitation to evaluating treatment effects was the lack of rigorous testing (i.e., no randomization, no control group). Furthermore, women were not screened in on the basis of their substance use, thus a major limitation in evaluating treatment effects.

TEAM-I. Findings indicated that alcohol, marijuana, and amphetamine usage decreased among all intervention groups from intake to 6-month follow-up; however, the MEI group showed highly significant $(p \leq 0.01)$ decreases when compared with the BI and control groups. The MEI intervention participants showed the most significant decreases in alcohol, marijuana, and amphetamine use frequency at 6-month follow-up, when compared with intake frequencies.

\section{Discussion}

This systematic review is the first to attempt a comprehensive identification and synthesis of the available evidence on interventions for reducing problematic substance use among transgender individuals. This review has clearly identified a large gap in the field of transgender health that needs attention and should be used as a call to action for efforts to address problematic substance use. The findings of this review elicit 


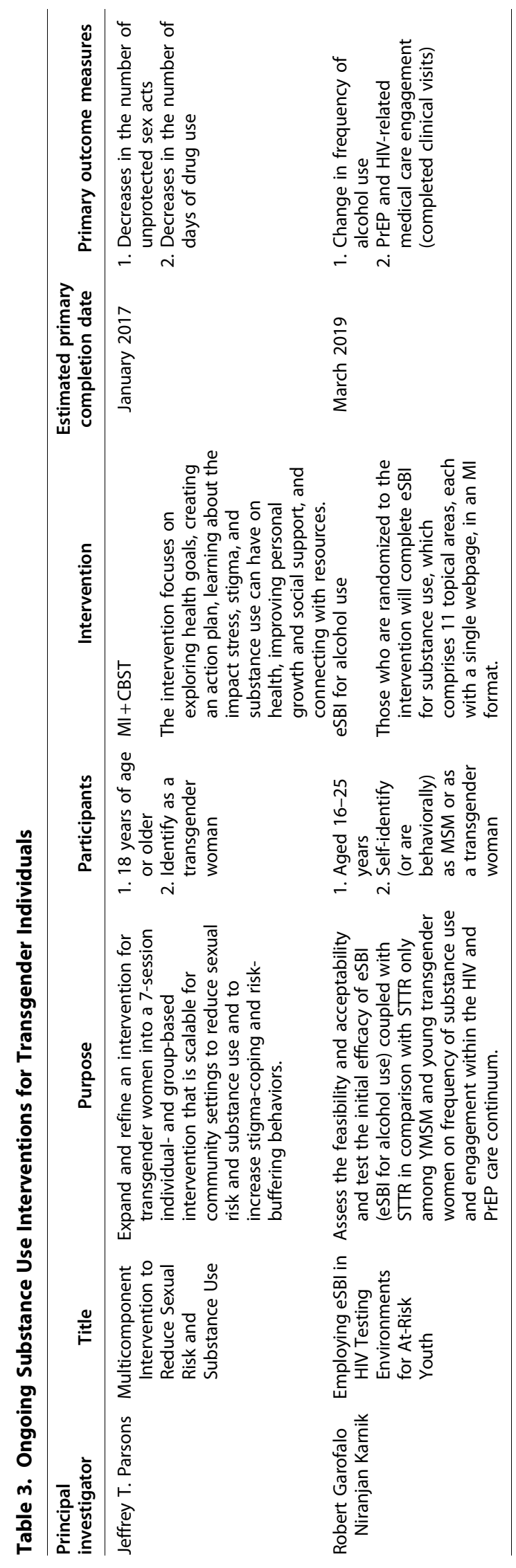

questions regarding how this major gap in the research literature has been facilitated and how to remedy it moving forward. In summary, there has been a lack of inclusion of transgender individuals in the current substance use research and intervention work; studies that do include transgender individuals have not collected gender identity or have not disaggregated findings to examine outcomes separately; and there is a lack of interventions developed specifically for transgender persons.

Perhaps one trend facilitating the gap between the need for treatment specific to transgender individuals and actual programs offering this care is the false belief that treatments do exist. Cochran et al. ${ }^{62}$ investigated if specialized substance use services existed for LGBT persons and found that of the 911 treatment listings in the United States and Puerto Rico, $11.8 \%$ of substance use programs indicated that they offer specialized services for sexual and gender minorities. However, the researchers conducted phone surveys and they revealed that $70.8 \%$ of these programs are the same services offered to the general population, and only $7.4 \%$ identified a service specifically tailored to the needs of LGBT individuals, which calculates to only eight programs of 911 substance use programs throughout the United States and Puerto Rico. False advertisement of treatment services that do claim specialized programs propagates the perception of having more services than are actually available and masks the real need for intervention development.

In addition, facilitating this gap is the issue that gender identity is rarely collected in substance use research, which is the most fundamental step to begin to address substance use intervention work. Between 2010 and 2011, the Institute of Medicine and US Department of Health and Human Services both set forth agendas to prioritize efforts for LGBT health research. ${ }^{1,63}$ Flentje et al. ${ }^{64}$ examined the reporting of sexual orientation and gender identity in the substance use literature during a recent time period before these national agendas were set forth (i.e., 2007). They then repeated this for a time period after these agendas (i.e., 2012) to see if the call for LGBT health research by national agencies moved the substance use field forward into efforts examining LGBT individuals. They found that in 2007, reporting of gender beyond the binary (i.e., male and female only) in the substance use literature happened between $0 \%$ and $1 \%$ in 2007 and was only raised to between $1.9 \%$ and $2.3 \%$ in 2012 . The omission of collecting gender identity in substance 
research is a missed opportunity to not only understand the well-being of transgender people, but it also means we are not learning about sex and gender differences that may be relevant to all people.

Beyond collecting gender identity, recruitment of gender minorities in substance use treatment studies is also crucial to be able to examine outcomes for these populations. For the literature that does collect and report on gender identity, it is commonly collapsed with sexual orientation, which is problematic because it prevents conclusions about the impact of substance use interventions for transgender individuals. For instance, five of the studies in this systematic review were excluded due to not reporting substance use outcomes separately for their transgender participants. There is a need to disaggregate findings for the $\mathrm{T}$ in LGBT, and also from more general populations, to develop effective evidence-based interventions specifically developed for transgender persons. Perhaps collapsing these groups together stems from the small number of transgender individuals within substance use intervention studies, pointing to the need for concentrated efforts in recruiting transgender individuals to be included within these studies.

In considering the way to move forward to address the identified gap of this review, it is critical to examine the body of research on substance use treatment for transgender individuals identified during this review, but excluded for methodological criteria. This literature has examined past experiences, barriers, acceptability, and associations of attending treatment. $6,37,39,62,65-72$ Important considerations from this research have emerged for future substance use intervention design. Numerous barriers for transgender individuals seeking treatment have been found that should be addressed when designing substance use programs, including unknowledgeable personnel in substance use treatments on trans-specific realities and experiences, treatment providers having negative attitudes toward transgender individuals, victimization (e.g., verbal, physical, and sexual abuse by other clients and staff), discrimination (e.g., being required to wear only clothes judged to be appropriate for their birth sex), and little formal education for staff regarding the needs of transgender people. ${ }^{13,37,68,71-76}$ Additionally, the current literature provides consistent themes in treatment recommendations for trans-specific substance use programs (Table 4).

One consistent recommendation for substance use interventions for transgender individuals is that using
Table 4. Overarching Themes in Recommendations for Substance Use Programs for Transgender Individuals

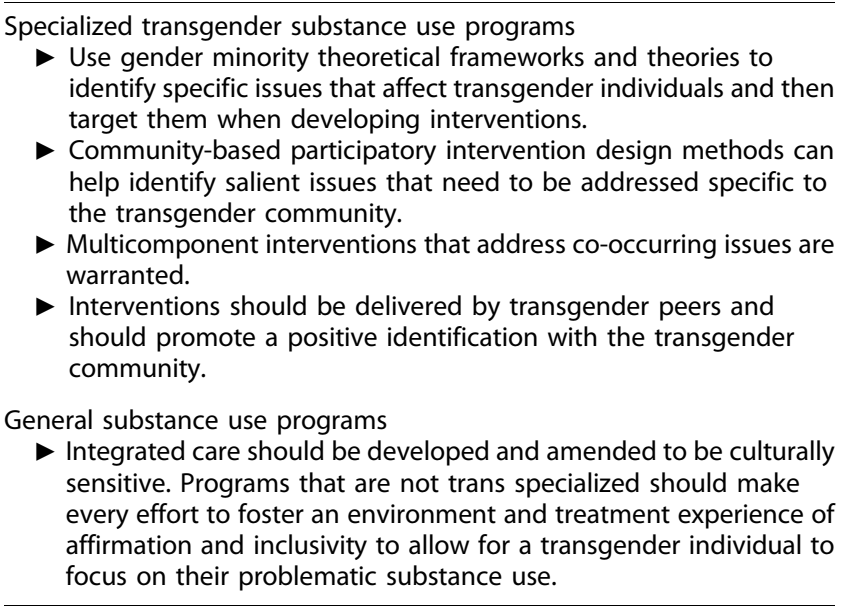

gender minority theoretical frameworks and theories (e.g., queer theory, transgender theory, and gender affirmation framework) can aid in identifying issues that affect transgender individuals specifically when developing interventions for substance use. ${ }^{39}$ Hendricks and Testa ${ }^{77}$ contextualized the Minority Stress Model for transgender individuals and recommend that the unique issues (e.g., transphobia, discrimination, and lack of support) that place transgender individuals at risk for health risk behaviors should be addressed directly in treatment. Others have also called for this theoretical approach in regard to substance use treatment for transgender individuals. ${ }^{13,39,41}$ Gender minority frameworks and theories can be supplemented by using community-based participatory research methods, as seen in the included intervention (TEAM-I). This would allow members and advocates of the community to confirm the needs of the transgender community that are posited by theories and frameworks and it may elicit new needs that have not been identified in the research.

A guideline endorsed by multiple scholars, and supported by the two included studies in this review, is that interventions should be delivered by transgender peers to allow for greater comfort in treatment and a more nuanced understanding of clients due to the unique experiences of transgender individuals. ${ }^{60,76,78}$ On a similar note, Hendricks and Testa ${ }^{77}$ suggest that clinicians should focus on promoting a positive identification with the transgender community as a means of buffering the negative effects of minority stressors, which is supported by the included studies in this systematic 
review. The Transgender Recovery Program has successfully implemented this within their residential substance use treatment program for transgender women. ${ }^{76}$ This intervention did not meet criteria for inclusion in the current review due to a lack of reporting substance use outcomes; however, they did report $81 \%$ retention in the program, noted as good compared with their general population program's retention of $60 \%$. Clients are encouraged to develop peer supports through their assigned Big Sister (i.e., another transgender woman) and through weekly small group meetings. The included study in this review also incorporated a community networking and empowerment domain within the intervention and also encouraged the women to socialize with each other in the program site. This group-level support provides a positive peer and community interaction that can foster resilience and recovery.

Addressing syndemic theory by building multicomponent interventions that address co-occurring issues is also warranted. $^{13,26,60,65,78,79}$ Targeting sexual behaviors is important for reducing the transmission of HIV and other sexually transmitted infections, but intervening on these alone may be insufficient in reducing new infections. The Positive Steps Program for women (both cisgender female and female identified) reported successful reductions in problematic substance use while targeting multiple facets of the HIV syndemic. ${ }^{79}$ This program did not meet criteria to be included in this review due to not separately reporting outcomes for transgender women, but warrants acknowledgement. This program is a 6-week residential chemical dependency program targeting substance use, sexual risk behaviors, and other person-level risk factors for HIV (e.g., gender identity and discrimination), which showed that alcohol and drug use declines from baseline to exit of program and to the 12-month follow-up along with a decline in psychological distress and sexual risk behaviors (i.e., condomless sex with an HIV-positive partner). The included programs, the TRANS project and TEAM-I, aimed to address multiple issues for transgender women such as HIV, drug use, discrimination, job seeking, relationships, and community involvement. By addressing the various epidemics that act multiplicatively to reinforce each other and facilitate substance use, it provides opportunity for long-term positive health outcomes and more holistic well-being.

Although specialized programs seem to be needed the most, there should be a greater awareness that these may not be easily and widely implemented due to various reasons such as the climate of transgender acceptance in some geographic locations ${ }^{80}$ or lack of financial resources to support these types of specialized programs. We should not only consider specialized programs but also substance use treatment providers should be challenged to meet the specific needs of transgender substance users within mainstream clinical settings to reduce further stigmatization and marginalization. ${ }^{39}$ Within these mainstream clinical settings, providers should be culturally competent to meet the needs of transgender people. ${ }^{13,81}$ Such integrated care should also be amended and developed to be culturally sensitive to transgender persons. For instance, although some trans-specific issues may be intertwined in an individual's substance use, providers need to be educated about identifying when gender issues are peripheral and not relevant to substance use treatment as to not overstate the entire transgender experience as a risk factor. ${ }^{82}$

Preventing discrimination by healthcare providers is also crucial for optimal treatment outcomes. Not specific to transgender individuals, negative attitudes have been found among healthcare professionals toward persons with problematic substance use and these attitudes have been associated with substandard treatment and care. ${ }^{83}$ Additionally, substance use treatment providers have been shown to have the least education about transgender individuals and have the greatest negativity toward them. ${ }^{68}$ Collectively, transgender individuals needing substance use treatment face exacerbated discrimination by providers for not only having problematic substance use but also for being transgender. Preventing discrimination within general substance use care from occurring produces a greater likelihood of successful treatment. Treatment staff should also be trained in transgender competence and sensitivity to make sure transgender inclusivity is not just written in a program's mission, but actually being practiced. ${ }^{41}$

Awareness of the unique needs of transgender people and sensitivity toward them are important in affirming an individual and providing an environment of acceptance. Examples include not restricting individuals' access to restrooms that are appropriate for their gender identity, not conflating transgender individuals with sexual minorities (lesbian, gay, etc.), allowing for gender presentation resources (e.g., makeup and clothing), sleeping arrangements or housing according to gender identity, allowance of hormone use, and using proper pronouns. ${ }^{37,72,76,84}$ In a sample of 
transgender individuals who had been in residential treatment for substance use, individuals who felt their environment was inclusive had more positive treatment experiences compared with those experiencing stigma and who left treatment prematurely. ${ }^{71}$ Programs that are not trans specialized should make every effort to foster an environment and treatment experience of affirmation and inclusivity to allow for a transgender individual to focus on their problematic substance use.

Limitations in the field should be noted when considering our findings. There may be transgender-specific substance use programs that are effective, but have not been integrated into the literature if they were not implemented as part of research. Researchers should consider examining current community programs to rigorously evaluate them to add them to the body of evidence. Notably, the literature regarding transgender individuals has been historically rooted in HIV prevention; given this, there have been several HIV prevention interventions for transgender individuals. These interventions may have had domains peripherally targeting substance use, but were not reported on due to the study's emphasis on HIV outcomes. The paucity of included studies only allowed for a collapsed examination of interventions for transgender women and transgender men. It is important to note that these groups have distinct experiences and may need disparate interventions. Both included studies were focused on transgender women, reflecting the lack of inclusion of transgender men in the current literature regarding transgender persons. Little is known about the substance use behaviors and risks associated with such use for transgender men; given this, there has been a recent call for action to include transgender men in research. ${ }^{11}$

This systematic review was only able to synthesize overall treatment recommendations, but it should be acknowledged that the transgender community is heterogeneous with subgroups having different intervention needs (e.g., transgender people of color). It should also be noted that there is a spectrum of gender identities (e.g., gender nonconforming and gender queer) and these individuals should not be collapsed in with transgender substance use research. Although some overlapping experiences occur given their gender minority status, these other groups also need specific attentions. At the level of systematic review, a limitation of this study is that there could be unidentified literature reporting on substance use intervention outcomes if outside of the chosen search strategy. Lim- itations notwithstanding, this review identifies major gaps that need immediate attention to move the transgender health field forward.

\section{Conclusions}

Although the treatment and intervention recommendations in the literature are important aspects of substance use research examining transgender individuals, moving toward designing and implementing culturally sensitive interventions are warranted and desired by the transgender community. ${ }^{17}$ Most recently, the NIAAA put forth an initiative to expand existing substance use research to focus on sexual and gender minority health ${ }^{85}$; this research agenda comes at a time of great need. Given this current sociopolitical climate of promoting psychosocial health among LGBT individuals, ${ }^{1}$ this offers a prime window of opportunity to research efficacious interventions toward problematic substance use and its psychological and social sequelae. Perhaps the most important conclusion of this review is that well-designed, theoretically informed culturally sensitive research focused on developing and rigorously testing interventions for substance use among transgender individuals is alarmingly scarce. The research regarding predictors, associations, barriers, and needs for transgender substance use treatment has been well documented; it is now time to design, implement, and disseminate interventions using this information to provide needed services to the transgender community.

\section{Acknowledgments}

This review was supported, in part, by the National Institutes of Health (NIH), including grants from the National Institute on Alcohol Abuse and Alcoholism (NIAAA) U24AA022000; PI: Operario, and the National Institute of Allergy And Infectious Diseases (NIAID) P30AI042853; PI: Cu-Uvin. The content is solely the responsibility of the authors and does not necessarily represent the official views of the NIAAA, NIAID, or NIH.

\section{Author Disclosure Statement}

No competing financial interests exist.

\section{References}

1. Institute of Medicine. The Health of Lesbian, Gay, Bisexual and Transgender People: Building a Foundation for Better Understanding. Committee on Lesbian, Gay Bisexual, and Transgender Health Issues and Research Gaps and Opportunities. Washington, DC: The National Academies Press, 2011 
2. World Health Organization. Substance abuse: terminology and classification. 2016. Available at www.who.int/substance_abuse/ terminology/en

3. National Institute on Alcohol Abuse and Alcoholism. Drinking Levels Defined. 2015. Available at www.niaaa.nih.gov/alcohol-health/overviewalcohol-consumption/moderate-binge-drinking

4. National Institute on Drug Abuse. Commonly Used Terms in Addiction Science. 2014. Available at www.drugabuse.gov/publications/mediaguide/glossary

5. American Psychological Association(APA). Statistical Manual of Mental Disorders: DSM-V. Washington, DC: APA, 2013.

6. Cochran BN, Cauce AM. Characteristics of lesbian, gay, bisexual, and transgender individuals entering substance abuse treatment. J Subst Abuse Treat. 2006;30:135-146.

7. Hughes T, Eliason M. Substance use and abuse in lesbian, gay, bisexual and transgender populations. J Prim Prevent. 2002;22: 263-298.

8. Garofalo R, Deleon J, Osmer E, et al. Overlooked, misunderstood and atrisk: exploring the lives and HIV risk of ethnic minority male-to-female transgender youth. J Adolesc Health. 2006;38:230-236.

9. Benotsch EG, Zimmerman R, Cathers L, et al. Non-medical use of prescription drugs, polysubstance use, and mental health in transgender adults. Drug Alcohol Depend. 2013;132:391-394.

10. Peacock $\mathrm{E}$, Andrinopoulos $\mathrm{K}$, Hembling J. Binge drinking among men who have sex with men and transgender women in San Salvador: correlates and sexual health implications. J Urban Health. 2015;92: 701-716.

11. Reisner SL, Murchison GR. A global research synthesis of HIV and STI biobehavioural risks in female-to-male transgender adults. Glob Public Health. 2016;11:866-887.

12. Santos GM, Rapues J, Wilson EC, et al. Alcohol and substance use among transgender women in San Francisco: prevalence and association with human immunodeficiency virus infection. Drug Alcohol Rev. 2014;33:287-295.

13. Wolf ECM, Dew BJ. Understanding risk factors contributing to substance use among MTF transgender persons. J LGBT Issues Couns. 2012;6: 237-256.

14. Lombardi E. Transgender health: a review and guidance for future research-Proceedings from the Summer Institute at the Center for Research on Health and Sexual Orientation, University of Pittsburgh. Int J Transgend. 2010;12:211-229.

15. Nuttbrock L, Bockting W, Rosenblum A, et al. Gender abuse, depressive symptoms, and substance use among transgender women: a 3-year prospective study. Am J Public Health. 2014;104:2199-2206.

16. Nemoto T, Operario D, Keatley J, et al. Social context of HIV risk behaviours among male-to-female transgenders of colour. AIDS Care. 2004;16:724-735.

17. Reisner SL, Gamarel KE, Dunham E, et al. Female-to-male transmasculine adult health: a mixed-methods community-based needs assessment. J Am Psychiatr Nurses Assoc. 2013;19:293-303.

18. Reisner SL, Pardo ST, Gamarel KE, et al. Substance use to cope with stigma in healthcare among U.S. female-to-male trans masculine adults. LGBT Health. 2015;2:324-332.

19. Rowe C, Santos GM, McFarland W, et al. Prevalence and correlates of substance use among trans female youth ages 16-24 years in the San Francisco Bay Area. Drug Alcohol Depend. 2015;147:160-166.

20. Super JT, Jacobson L. Religious abuse: implications for counseling lesbian, gay, bisexual, and transgender individuals. J LGBT Issues Couns. 2011;5:180-196.

21. Meyer IH. Prejudice, social stress, and mental health in lesbian, gay, and bisexual populations: conceptual issues and research evidence. Psychol Bull. 2003;129:674-697.

22. Herbst J, Jacobs E, Finlayson T, et al. Estimating HIV prevalence and risk behaviors of transgender persons in the United States: a systematic review. AIDS Behav. 2008;12:1-17.

23. Clements-Nolle K, Marx R, Guzman R, et al. HIV prevalence, risk behaviors, health care use, and mental health status of transgender persons: implications for public health intervention. Am J Public Health. 2001;91:915-921.

24. Reback CJ, Fletcher JB. HIV prevalence, substance use, and sexual risk behaviors among transgender women recruited through outreach. AIDS Behav. 2014;18:1359-1367.
25. Singer M, Clair S. Syndemics and public health: reconceptualizing disease in bio-social context. Med Anthropol Q. 2003;17:423-441.

26. Operario D, Nemoto T. HIV in transgender communities: syndemic dynamics and a need for multicomponent interventions. J Acquir Immune Defic Syndr. 2010;55:S91-S93.

27. Brennan J, Kuhns LM, Johnson AK, et al. Syndemic theory and HIVrelated risk among young transgender women: the role of multiple, co-occurring health problems and social marginalization. Am J Public Health. 2012;102:1751-1757.

28. Chakrapani V, Newman PA, Shunmugam M, et al. Syndemics of depression, alcohol use, and victimisation, and their association with HIVrelated sexual risk among men who have sex with men and transgender women in India. Glob Public Health. 2017;12:250-265.

29. Reisner SL, White Hughto JM, Pardee D, et al. Syndemics and gender affirmation: HIV sexual risk in female-to-male trans masculine adults reporting sexual contact with cisgender males. Int J STD AIDS. 2016;27:955-966.

30. Centers for Disease Control and Prevention. HIV and Substance Use in the United States. 2015. Available at www.cdc.gov/hiv/risk/ substanceuese.html

31. De Santis JP. HIV Infection risk factors among male-to-female transgender persons: a review of the literature. J Assoc Nurs AIDS Care. 2009;20:362-372.

32. Nemoto T, Operario D, Keatley J, et al. HIV risk behaviors among male-tofemale transgender persons of color in San Francisco. Am J Public Health. 2004;94:1193-1199.

33. Hotton AL, Garofalo R, Kuhns LM, et al. Substance use as a mediator of the relationship between life stress and sexual risk among young transgender women. AIDS Educ Prev. 2013;25:62-71.

34. Benotsch EG, Zimmerman RS, Cathers L, et al. Non-medical use of prescription drugs and HIV risk behaviour in transgender women in the Mid-Atlantic region of the United States. Int J STD AIDS. 2016;27:776-782.

35. Herrera MC, Konda KA, Leon SR, et al. Impact of alcohol use on sexual behavior among men who have sex with men and transgender women in Lima, Peru. Drug Alcohol Depend. 2016;161:147-154.

36. Centers for Disease Control and Prevention. HIV Among Transgender People. 2015. Available at www.cdc.gov/hiv/group/gender/transgender

37. Lombardi E, van Servellen G. Building culturally sensitive substance use prevention and treatment programs for transgendered populations. J Subst Abuse Treat. 2000;19:291-296.

38. Craft EM, Mulvey KP. Addressing lesbian, gay, bisexual, and transgender issues from the inside: one federal agency's approach. Am J Public Health. 2001;91:889-891.

39. Stevens S. Meeting the substance abuse treatment needs of lesbian, bisexual and transgender women: implications from research to practice. Subst Abuse Rehabil. 2012;3:27-36.

40. The Transgender Substance Abuse Treatment Policy Group of the San Francisco Lesbian Gay, Bisexual, and Transgender Task Force. Transgender Protocol: Treatment Services Guidelines for Substance Abuse Treatment Providers. San Francisco, CA: Transgender Substance Abuse Treatment Policy Group, 1995.

41. Sevelius JM, Reznick OG, Hart SL, et al. Informing interventions: the importance of contextual factors in the prediction of sexual risk behaviors among transgender women. AIDS Educ Prev. 2009;21:113-127.

42. Nemoto T, Operario D, Keatley J. Health and social services for male-tofemale transgender persons of color in San Francisco. Int J Transgend. 2005;8:5-19.

43. Dutra L, Stathopoulou G, Basden SL, et al. A meta-analytic review of psychosocial interventions for substance use disorders. Am J Psych. 2008;165:179-187.

44. Magill M, Ray LA. Cognitive-behavioral treatment with adult alcohol and illicit drug users: a meta-analysis of randomized controlled trials. J Stud Alcohol Drugs. 2009;70:516-527.

45. Tait RJ, Hulse GK. A systematic review of the effectiveness of brief interventions with substance using adolescents by type of drug. Drug Alcohol Rev. 2003;22:337-346.

46. Carney T, Myers B. Effectiveness of early interventions for substanceusing adolescents: findings from a systematic review and meta-analysis. Subst Abuse Treat Prev Policy. 2012;7:1.

47. Williams RJ, Chang SY. A comprehensive and comparative review of adolescent substance abuse treatment outcome. Clin Psychol. 2000;7:138-166. 
48. Tanner-Smith EE, Wilson SJ, Lipsey MW. The comparative effectiveness of outpatient treatment for adolescent substance abuse: a metaanalysis. J Subst Abuse Treat 2013;44:145-158.

49. Waldron $\mathrm{HB}$, Turner CW. Evidence-based psychosocial treatments for adolescent substance abuse. J Clin Child Adolesc Psychol. 2008;37:238261.

50. Gil AG, Wagner EF, Tubman JG. Culturally sensitive substance abuse intervention for Hispanic and African American adolescents: empirical examples from the Alcohol Treatment Targeting Adolescents in Need (ATTAIN) Project. Addiction. 2004;99:140-150.

51. Schonfeld L, King-Kallimanis BL, Duchene DM, et al. Screening and brief intervention for substance misuse among older adults: the Florida BRITE project. Am J Public Health. 2010;100:108-114.

52. Gilchrist LD, Schinke SP, Trimble JE, et al. Skills enhancement to prevent substance abuse among American Indian adolescents. Int J Addict. 1987;22:869-879.

53. Paul JP, Barrett DC, Crosby GM, et al. Longitudinal changes in alcohol and drug use among men seen at a gay-specific substance abuse treatment agency. J Stud Alcohol. 1996;57:475-485.

54. Shamseer L, Moher D, Clarke M, et al. Preferred reporting items for systematic review and meta-analysis protocols (PRISMA-P) 2015: elaboration and explanation. BMJ. 2015;349.

55. Rösner S, Willutzki R, Zgierska A. Mindfulness-based interventions for substance use disorders. Cochrane Database Syst Rev. 2015.

56. Klimas J, Tobin H, Field C-A, et al. Psychosocial interventions to reduce alcohol consumption in concurrent problem alcohol and illicit drug users. Cochrane Database Syst Rev. 2014.

57. First MB, Williams JBW, Karg RS, et al. Structured Clinical Interview for DSM-5-Research Version (SCID-5 for DSM-5, Research Version; SCID-5RV). Arlington, VA: American Psychiatric Association, 2015.

58. Wray TB, Grin B, Dorfman L, et al. Systematic review of interventions to reduce problematic alcohol use in men who have sex with men. Drug Alcohol Rev. 2015;n/a.

59. Baral SD, Poteat T, Strömdahl S, et al. Worldwide burden of HIV in transgender women: a systematic review and meta-analysis. Lancet Infect Dis. 2013;13:214-222.

60. Nemoto T, Operario D, Keatley J, et al. Promoting health for transgender women: Transgender Resources and Neighborhood Space (TRANS) program in San Francisco. Am J Public Health. 2005;95:382-384.

61. Nemoto T, Iwamoto M, Eilkhani E, et al. Substance Abuse and HIV Prevention Intervention Study for Transgender Women of Color. 141st American Public Health Association (APHA) annual meeting, 2013, Boston, MA.

62. Cochran BN, Peavy KM, Robohm JS. Do specialized services exist for LGBT individuals seeking treatment for substance misuse? A study of available treatment programs. Subst Use Misuse. 2007;42:161-176.

63. United States Department of Health and Human Services. Lesbian, Gay, Bisexual, and Transgender Health. 2010. Available at www.healthypeople.gov/2020/topics-objectives/topic/lesbian-gaybisexual-and-transgender-health (accessed on March 25, 2016).

64. Flentje A, Bacca CL, Cochran BN. Missing data in substance abuse research? Researchers' reporting practices of sexual orientation and gender identity. Drug Alcohol Depend. 2015;147:280-284.

65. Keuroghlian AS, Reisner SL, White JM, et al. Substance use and treatment of substance use disorders in a community sample of transgender adults. Drug Alcohol Depend. 2015;152:139-146.

66. Lombardi E. Substance use treatment experiences of transgender/ transsexual men and women. J LGBT Health Res. 2007;3:37-47.

67. Flentje A, Heck NC, Sorensen JL. Characteristics of transgender individuals entering substance abuse treatment. Addict Behav. 2014;39:969-975.

68. Eliason MJ. Substance abuse counsellor's attitudes regarding lesbian, gay, bisexual, and transgendered clients. J Subst Abuse. 2000;12:311-328.

69. Cochran BN, Peavy KM, Cauce AM. Substance abuse treatment providers' explicit and implicit attitudes regarding sexual minorities. J Homosex. 2007;53:181-207.

70. Brown $\mathrm{SE}$, Vagenas $\mathrm{P}$, Konda KA, et al. Men who have sex with men in Peru: acceptability of medication-assisted therapy for treating alcohol use disorders. Am J Mens Health. 2015. [Epub ahead of print]; DOI: 10.1177/1557988315576775.

71. Lyons T, Shannon K, Pierre L, et al. A qualitative study of transgender individuals' experiences in residential addiction treatment settings: stigma and inclusivity. Subst Abuse Treat Prev Policy. 2015;10:17.
72. Senreich $E$. The substance abuse treatment experiences of a small sample of transgender clients. J Soc Work Pract Addict. 2011;11:295299.

73. Bockting WO, Robinson BE, Rosser BRS. Transgender HIV prevention: a qualitative needs assessment. AIDS Care. 1998;10:505-525.

74. Eliason MJ, Hughes T. Treatment counselor's attitudes about lesbian, gay, bisexual, and transgendered clients: urban vs. rural settings. Subst Use Misuse. 2004;39:625-644.

75. Delany-Moretlwe S, Cowan FM, Busza J, et al. Providing comprehensive health services for young key populations: needs, barriers and gaps. J Int AIDS Soc. 2015;18:19833.

76. Oggins J, Eichenbaum J. Engaging transgender substance users in substance use treatment. Int J Transgend. 2002;6:1.

77. Hendricks ML, Testa RJ. A conceptual framework for clinical work with transgender and gender nonconforming clients: an adaptation of the Minority Stress Model. Prof Psychol Res Pr. 2012;43:460-467.

78. Nemoto T, Sausa LA, Operario D, et al. Need for HIV/AIDS education and intervention for MTF transgenders: responding to the challenge. J Homosex. 2006;51:183-202.

79. Harris E, Kiekel P, Brown K, et al. A multicultural approach to HIV prevention within a residential chemical dependency treatment program: the Positive Steps Program. J Evid Based Soc Work. 2010;7:58-68.

80. Willging CE, Salvador M, Kano M. Pragmatic help seeking: how sexual and gender minority groups access mental health care in a rural state. Psychiatr Serv. 2006;57:871-874.

81. Polak K, Haug NA, Drachenberg HE, et al. Gender Considerations in Addiction: Implications for Treatment. Curr Treat Opt Psych. 2015;2:326338.

82. Sperber J, Landers S, Lawrence S. Access to health care for transgendered persons: results of a needs assessment in Boston. Int J Transgend. 2005;8:75-91.

83. van Boekel LC, Brouwers EPM, van Weeghel J, et al. Stigma among health professionals towards patients with substance use disorders and its consequences for healthcare delivery: systematic review. Drug Alcohol Depend. 2013;131:23-35.

84. Nuttbrock LA. Culturally competent substance abuse treatment with transgender persons. J Addict Dis. 2012;31:236-241.

85. National Institute on Alcohol Abuse and Alcoholism (NIAAA). NIAAA Director's Report on Institute Activities to the 140th Meeting of the National Advisory Council on Alcohol Abuse and Alcoholism. Bethesda, MD: NIAAA, 2015.

86. Dunckley CM. Julius. J Contin Educ Health Prof. 2005;25:127-128.

87. Finlon C. Substance abuse in lesbian, gay, bisexual, and transgender communities. J Gay Lesbian Soc Serv. 2002;14:109-116.

88. Gelaude DJ, Sovine ML, Swayzer R, et al. HIV prevention programs delivered by community-based organizations to young transgender persons of color: lessons learned to improve future program implementation. Int J Transgend. 2013;14:127-139.

89. Gilbert L, Raj A, Hien D, et al. Targeting the SAVA (Substance Abuse, Violence, and AIDS) syndemic among women and girls: a global review of epidemiology and integrated interventions. J Acquir Immune Defic Syndr. 2015;69(Suppl 2):S118-S127.

90. Heck NC, Flentje A, Cochran BN. Offsetting risks: high school gay-straight alliances and lesbian, gay, bisexual, and transgender (LGBT) youth. School Psychol Quart. 2011;26:161-174.

91. Hellman RE, Klein E, Drescher J. A program for lesbian, gay, bisexual, and transgender individuals with major mental illness. J Gay Lesbian Ment Health. 2004;8:67-82.

92. Jordan KM. Substance abuse among gay, lesbian, bisexual, transgender, and questioning adolescents. School Psychol Rev. 2000;29:201.

93. Klein AA, Ross BL. Substance use and mental health severity among LGBTQ individuals attending Minnesota model-based residential treatment. J Gay Lesbian Soc Serv. 2014;26:303-317.

94. Krishnan A, Ferro EG, Weikum D, et al. Communication technology use and $\mathrm{mHealth}$ acceptance among HIV-infected men who have sex with men in Peru: implications for HIV prevention and treatment. AIDS Care. 2015;27:273-282.

95. Medeiros DM, Seehaus M, Elliott J, et al. Providing mental health services for LGBT teens in a community adolescent health clinic. J Gay Lesbian Psychother. 2004;8:83-95.

96. Patel VV, Masyukova M, Sutton D, et al. Social media use and HIV-related risk behaviors in young black and latino gay and bi men and 
transgender individuals in New York City: implications for online interventions. J Urban Health. 2016;93:388-399.

97. Pettifor A, Nguyen NL, Celum C, et al. Tailored combination prevention packages and PrEP for young key populations. J Int AIDS Soc. 2015;18:19434.

98. Ritter A. New Australian lesbian, gay, bisexual and transgender research - and the need for more. Drug Alcohol Rev. 2015;34:347-348.

99. Senreich E. Inviting the significant other of LGBT clients into substance abuse treatment programs: frequency and impact. Contemp Fam Ther. 2010;32:427-443.

100. Senreich E. Differences in outcomes, completion rates, and perceptions of treatment between white, black, and Hispanic LGBT clients in substance abuse programs. J Gay Lesbian Mental Health. 2010;14:176-200.

101. Silvestre A, Beatty RL, Friedman MR. Substance use disorder in the context of LGBT health: a social work perspective. Soc Work Public Health. 2013;28:366-376.

102. Warren BE. Sex, truth, and videotape: HIV prevention at the gender identity project in New York City. Int J Transgend. 1999;3:1.

103. Ziegler PP. Treating gay, lesbian, bisexual and transgender professionals with addictive disease. J Gay Lesbian Psychother. 2000;3:59-68.

104. Goldbach JT, Holleran Steiker LK. An examination of cultural adaptations performed by LGBT-identified youths to a culturally grounded, evidence-based substance abuse intervention. J Gay Lesbian Soc Serv. 2011;23:188-203.

105. Goyal S, Deb KS, Elawadhi D, et al. Substance abuse as a way of life in marginalized gender identity disorder: a case report with review of $\mathrm{In}$ dian literature. Asian J Psychiatr. 2014;12:160-162.

106. Hicks D. The importance of specialized treatment programs for lesbian and gay patients. J Gay Lesbian Psychother. 2000;3:81-94.

107. Maguen S, Shipherd JC, Harris HN. Providing culturally sensitive care for transgender patients. Cognitive Behav Pract. 2005;12:479-490.

108. Reback CJ, Lombardi EL. HIV risk behaviors of male-to-female transgenders in a community-based harm reduction program. Int J Transgend. 1999;3:1.

109. Maxwell S, Shinderman MS. Use of naltrexone in the treatment of alcohol use disorders in patients with concomitant major mental illness. J Addict Dis. 2000;19:61-69.

110. Rowan NL, Faul AC. Gay, lesbian, bisexual, and transgendered people and chemical dependency: exploring successful treatment. J Gay Lesbian Soc Serv. 2011;23:107-130.

111. Schwinn TM, Thom B, Schinke SP, et al. Preventing drug use among sexual-minority youths: findings from a tailored, web-based intervention. J Adolesc Health. 2015;56:571-573.

112. Yu J, Appel $P$, Rogers $M$, et al. Integrating intervention for substance use disorder in a healthcare setting: practice and outcomes in New York City STD clinics. Am J Drug Alcohol Abuse. 2016:42:32-38.

113. Senreich E. Demographic, background, and treatment factors that affect gay and bisexual clients in substance abuse programs. J LGBT Issues Counsel. 2009;3:177-197.

114. Allison SM, Adams D, Klindera KC, et al. Innovative uses of communication technology for HIV programming for men who have sex with men and transgender persons. J Int AIDS Soc. 2014;17:19041.

Cite this article as: Glynn TR, van den Berg JJ (2017) A systematic review of interventions to reduce problematic substance use among transgender individuals: a call to action, Transgender Health 2:1, 45-59, DOI: 10.1089/trgh.2016.0037.

$\begin{aligned} & \text { Abbreviations Used } \\ & \mathrm{BI}=\text { brief intervention } \\ & \mathrm{CBST}=\text { cognitive-behavioral skills training } \\ & \mathrm{eSBI}=\text { electronic screening and brief intervention } \\ & \mathrm{LGBT}=\text { lesbian, gay, bisexual, and transgender } \\ & \mathrm{MEI}=\text { motivational enhancement intervention } \\ & \mathrm{MI}=\text { motivational interviewing } \\ & \mathrm{NIAAA}=\text { National Institute on Alcohol Abuse and Alcoholism } \\ & \mathrm{NIH}=\text { National Institutes of Health } \\ & \mathrm{PrEP}=\text { pre-exposure prophylaxis } \\ & \mathrm{RCT}=\text { randomized control trial } \\ & \mathrm{STTR}=\text { seek, test, treat, and retain } \\ & \mathrm{TEAM} \mathrm{I}=\text { Transgender Empowerment and Motivational } \\ & \mathrm{TRANS}=\text { Interviewing } \\ & \mathrm{YMSM}=\text { young men who have sex with men }\end{aligned}$




\section{Appendix A1. Search Terms for Electronic Databases}

Population: transgender

transgender OR transgend* OR trans-gend* OR transsex* OR trans-sex* OR transex* OR "sex reassign*" OR "sex change" OR "gender reassign*" OR "gender change" OR intersex* OR "gender identity disorder" OR "MTF" OR "male to female transgender" OR "male-to-female transgender" OR "male to female transsexual" OR "male-to-female transsexual" OR "male to female transexual" OR "male-to-female transexual" OR "FTM" or "female to male transgender" OR "female-to-male transgender" OR "female to male transsexual" OR "female-to-male transsexual" OR "female to male transexual" OR "female-to-male transexual" OR transvest* OR "travesty" OR "koti" OR "kothi*" OR "hijra" OR "mahuvahine" OR "mahu" OR "waria" OR "banci" OR "calabai" OR "kedie" OR "kawekawe" OR "wan du" OR "bissu" OR "katoey" OR "kathoey" OR "pet tee sam" OR "phuying praphet song" OR "sao praphet song" OR "nang fa jam leng" OR "ork-sao" OR "tut" OR "ladyboy" OR "ladyman" OR "meti" OR "cross dress" OR "acault" OR "bayot" OR "bantut" OR "bakla" OR "bian xing ren" OR "bong lo" OR "nadleehi" OR "berdache" OR "xanith" OR genderqueer OR "gender queer" OR "gender nonconforming" OR "gender non-conforming"

\section{Outcome: substance use}

"substance use disorder" OR "alcohol use disorder" OR "substance use disorders" OR "alcohol use disorders" OR misuse OR dependen* OR abuse OR substance OR polysubstance OR drug* OR polydrug OR illicit OR illegal OR abstin* OR abstain* OR abuse* OR addict* OR overdose OR withdrawal* OR disorder* OR cocaine OR crack OR speed OR heroin OR amphetamine* OR "designer drugs" OR narcotic* OR "designer drug" OR methamphetamine* OR crystal OR meth OR "off-label use" OR prescription OR opioid* OR opiate* OR opium OR ecstasy OR mdma OR hallucinogen* OR Isd OR pcp OR ketamine OR acid OR "club drugs" OR "club drug" OR downer* OR sedative* OR barbiturate* OR upper* OR methadone OR morphine OR marijuana OR marihuana OR cannabis OR weed OR pot OR "hash oil*" OR hashish OR alcohol OR drink* OR binge OR intoxic*

\section{Appendix A2. Search Strategies for Ongoing Studies}

Resource URL

ClinicalTrials.gov http://clinicaltrials.gov

European Union Clinical Trials Register www.clinicaltrialsregister.eu

World Health Organization International Clinical Trials Registry Platform Search Portal http://apps.who.int/trialsearc
Search terms

Transgender OR transsexual

Transgender OR transsexual

Transgender OR transsexual

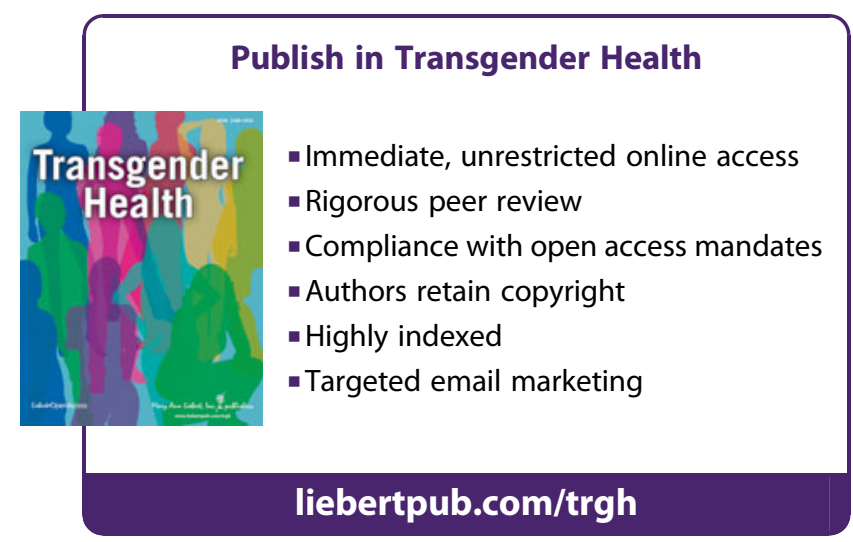

